\title{
Calculating the energy of fullerenes
}

\author{
So far, the experimentalists have had the most fun from fullerenes, but now the theorists are catching up. Not before \\ time, for they have to live down the shame of not having predicted them.
}

THAT the discovery of such molecules as $\mathrm{C}_{60}$ has been a surprise goes without saying. That they should be made so crudely, for example by collecting the soot from a carbon arc and not by a team effort in some organic chemistry synthesis laboratory, is only part of the tale. That truncated icosahedral cages of carbon atoms should be stable relative to, say, flattened sheets is another. That they should also be chemically akin to alkenes more than to benzene is yet another. And since the discovery of the simplest fullerenes, larger cage structure made only of carbon have been found, as have been the famous nanotubes which may yet become (when filled with suitable metallic atoms) the first electrically conducting nano-wires and the still more puzzling structures consisting of a small number of concentric shells of carbon atoms held together as if there were an onion. Sceptics must be forgiven for asking where it will all end

Inevitably, the search for new forms of this unexpected allotrope of carbon has been matched in intensity only by the attempts of theorists to calculate the laboratory features of the fullerenes so far observed. The simplest tasks are easily done. Electronically, $\mathrm{C}_{60}$ is the three-dimensional analogue of benzene, so that the electron states can be calculated by a simple extension of material found in many text-books. The thermodynamic properties of the crystalline form, and in particular the degree to which rotation of the cages is reduced with decreasing tenperature, can be estimated, most accurately by molecular dynamics. It remains a puzzle that interstitial potassium ions appear to turn the $\mathrm{C}_{60}$ solid into a superconductor, but even the superconductivity of the copper-oxide ceramics remains obscure except to those who must be supposed to understand their own theories.

Similar tasks have been carried out for larger fullerene molecules, if and when there is some guidance about the symmetry of the molecular structure (or the molecule is small enough for the alternatives to be enumerated). Meanwhile, it has ceased to be a cause for wonder that the supposed baseline of the energetic stability of carbon atoms in bulk - the electronically conjugated carbon atoms in a sheet of graphite - so readily curls up into icosahedral molecules. An infinite sheet of carbon atoms may be the most stable form there can be, but a finite piece of the same structure has so many unrequited or "dangling" chemical bonds that the energy of bond-bending when forming even a
$\mathrm{C}_{60}$ cage is far outdone by the energy of forming the covalent bonds that hold the structure together.

In passing, it is not irreverent to recall that the existence of $\mathrm{C}_{60}$ was almost entirely overlooked by theorists until the molecule showed up in carbon soot. For the past quarter of a century, people have been working with conjugated electron systems whose complexity has steadily increased. Yet the suggestions that a spheroidal form of graphite might be stable were few and little heeded before its discovery in 1985. E. Osawa proposed in 1970 that $C_{60}$ might be stable, and the Soviets D.A. Bochvar and E.G. Gal'pern went so far as to perform molecular-orbital calculations in 1973; while at the University of California at Los Angeles, Orville Chapman attempted the very synthesis alluded to earlier. But somehow, the attractive idea of an icosahedral cage molecule failed to spread wider roots.

Whatever the explanation, and despite the variety of molecularly distinct forms (isomers included) of the fullerenes found so far, there seems still to be a long way to go before this surprising ode is worked out. That at least is the inference from the work of J. Bernholc and two colleagues at the University of North Carolina (Maiti, A., Brabec, C. \& Bernholc, J. Phys. Rev. Lett. 70, 3023; 17 May 1993). Although there has been a spate of attempts to calculate the energy of particular fullerenes in the past four years, Bernholc and his colleagues reach some startling conclusions about fullerenes not yet made by a refreshing mixture of verbal argument with calculation. (More formally, they call it "scaling arguments and explicit energetic considerations".) The calculation part consists of molecular dynamics in which spurious energy minima (corresponding to fullerene cages with defects) are avoided by imposing an artificial symmetry on the system.

Perhaps the most startling result is not a result at all, but a kind of laboratory notebook entry; the calculations have been extended to cage-like fullerenes with more than 6,000 identical carbon atoms in order that their energy can be compared with that of the onion-structured isomers, with a few concentric cages rather than one large cage. The surprising result is that the most stable (or least energetic form) of the single-shelled structure is always a polyhedron (so that puckered shells are unfavoured aberrations). Among isomers for a fullerene of specified even-integer rank, the most stable form is always that in which there are exactly twelve pentagons. (Fullerenes of odd rank are intrinsically odd; they cannot satisfy the condition that there should be exactly three bonds at each vertex.) Icosahedral symmetry is also an advantage.

Otherwise, the outcome of the calculations is so regular that the authors are able to fit it by a graph for the energy per carbon atom in the shell which is a smoothly declining function of the number of atoms and which appears to be accurate to a few parts in 10,000 for shells containing up to 10,000 carbon atoms. The snag is simply that the energy of the lowest possible state of a shell of specified size may not be very different from others only slightly less stable. That is why the special symmetry of the theoretical ground state may not be that found in crystals of these super-fullerene molecules - if the materials themselves are ever found.

Armed with information, the business of calculating the onion-structures is then straightforward. It is merely necessary to arrange that all shells will indeed fit within the next largest as if it were a Russian doll slipped inside the next largest in the sequence. But the onion structure become stable relative to the single shell only when the number of atoms is so great that the mutual attractive energy of the concentric layers exceeds that associated with the extra curvature of the surfaces.

None of this is earthshaking. On the contrary (and apart from the rule that the most stable full recognizable shape must be an open question, especially if there is so little difference between the energy of the ground state and those next to it in stability).

In any case, there is plenty of work on fullerenes to occupy the theorists for some time. The electronic structure of molecules such as these which appear to be deformable at low energetic cost seems an interesting problem. How the geometric constraints might influence the electronic band structure may be a still more subtle issue. Carbon nanotubes, for example, are predicted to transform from insulators into semiconductors merely by the variation of the helical twist in their chicken-wire structure. And with the further possibility of tuning the electronical and mechanical properties by stuffing these hollow structures with metals, or even by doping the carbon sheets themselves with atoms such as boron, the permutations available for theoretical exploration seem endless. (It seems unlikely that the theorists will be caught napping a second time.)

John Maddox 